\title{
O Uso de Fatias de Tecido Cerebral ("Brain Slices") no Estudo da Epilepsia do Lobo Temporal
}

\author{
E. R. G. Sanabria*, F. A. Scorza* \& E. A. Cavalheiro**
}

\begin{abstract}
RESUMO
A epilepsia do lobo temporal (ELT) é a forma mais comum de epilepsia em adultos e responsável por um grande número de casos de refratariedade farmacológica a drogas anticonvulsivantes convencionalmente usadas, sendo que estes pacientes apresentam uma sintomatologia clínica caracterizada por crises parciais complexas que se originam nas estruturas mesiais do lobo temporal, as quais săo freqüentemente de difícil controle. A ELT é uma entidade amplamente estudada, que apresenta uma estreita relação entre a formação hipocampal e a atividade epilética. Diante disso, diversos modelos experimentais de epilepsia do lobo temporal têm sido desenvolvidos para um melhor estudo dos possiveis mecanismos envolvidos na epileptogênese do hipocampo. Uma técnica recentemente aplicada nos estudos destes mecanismos básicos neuronais é a técnica de "slices" (fatias vivas) do hipocampo, sendo este um dos procedimentos mais importantes no estabelecimento da neurociência a nivel mundial. Os objetivos principais dos estudos eletrofisiológicos em "slices" cerebrais são a descrição das propriedades celulares e biofisicas dos neurônios epiléticos, como também a avaliação da funçăo sináptica (fenómenos plásticos) e de sistemas de neurotransmissores, podendo auxiliar na determinação dos eventos patoiógicos celulares, sinápticos e moleculares (canais iônicos) envolvidos com os mecanismos de propagaçāo e sincronização da atividade epileptiforme.
\end{abstract}

\section{UNITERMOS}

"Slices" de Hipocampo; Eletrofisiologia; Epilepsia do lobo temporai; Esclerose hipocampal.

Pós-graduandos em Neurologia/ Neurociências -UNIFESP-EPM.

* Professor Títular e Chefe da Disciplina de Neurologia Experimental. Departamento de Neurologia Clinica e Neurocirurgia. Universidade Federal de São Paulo-Escola Paulista de Medicina.

\section{INTRODUÇÃO}

A epilepsia do lobo temporal (ELT) é a forma mais comum de epilepsia em adultos $(40 \%)$, de início na infância tardia ou adolescência ${ }^{49}$. ELT é una entidade amplamente estudada, que apresenta uma estreita relação entre a formação hipocampal e a atividade epilética. Diversos modelos experimentais de epilepsia do lobo temporal têm sido desenvolvidos para um melhor estudo dos possíveis mecanismos envolvidos na epileptogênese do hipocampo ${ }^{11,27,45.55}$. Uma técnica recentemente aplicada nos estudos destes mecanismos básicos neuronais é a técnica de "slices" (fatias vivas) do hipocampo. Este procedimento oferece inúmeras oportunidades de pesquisa para anatomistas, fisiologistas, farmacologistas, biologistas moleculares, bioquímicos e biofísicos no estudo dos mecanismos básicos das epilepsias, especificamente no estudo da epilepsia do lobo temporal. Esta técnica tem sido usada em modelos experimentais, assim como em tecido humano obtido nas cirurgias de pacientes epiléticos submetidos ao tratamento cirúrgico.

\section{HISTÓRICO}

O desenvolvimento da técnica de "brain slices" é um dos procedimentos mais importantes no estabelecimento da neurociência a nível mundial. As preparações de "brain slices" foram desenvolvidas por McIlwain e colaboradores na década de 50, com o entuito de se estudar a bioquímica do sistema nervoso centra ${ }^{54}$. A notoriedade desta técnica consiste em demonstrar que os neurônios podem ser mantidos em estado saudável para poderem ser estudados. Mais tarde, este mesmo grupo de neurocientistas adotaram técnicas de eletrofisiologia que consolidaram estes estudos nos "slices". Em 1966, Yamamoto e McIlwain descreveram a primeira preparação sinapticamente viável ${ }^{107}$. A era dos "brain slices" teve início realmente em 1957 , quando 
Luh Li e Mcllwain mostraram que "slices" do neocórtex do cérebro de cobaias e gatos mantêm de forma estável o potencial de membrana em repouso ${ }^{44}$. Posteriormente, foram criadas novas técnicas de aplicação de drogas e substâncias farmacologicamente ativas nos "slices" Curiosamente, uma das substâncias que estes autores selecionaram para estudar foi o L-glutamato, fato marcante e de particular relevância nas subsequientes investigações em neurociência ${ }^{14}$. A técnica de "slices" oferece a vantagem de se poder controlar a viabilidade do meio, o pH e aplicação de drogas, além de manter a estabilidade física e metabólica dos mesmos ${ }^{14}$. Por outro lado, o tecido é completamente isolado do influxo neural e hormonal ${ }^{36}$. Modernas técnicas eletrofisiológicas, através de registros intracelulares, extracelulares e "patch-clamp" foram posteriormente desenvolvidas ${ }^{24.32 .76}$. Recentemente, os "slices" têm sido utilizados para estudos imunocitoquímicos, de hibridização in situ ${ }^{1 \prime \prime}$ e de radicais livres ${ }^{68}$. Uma nova técnica avançada são os "slices" de longa duração, ou cultivos de "slices" (normalmente os "slices" são viáveis por 5 a 7 horas), permitindo um estudo mais completo e duradouro $^{18}$. Todos estes fatos levaram os pesquisadores a utilizar esta técnica nas investigações dos mecanismos eletrofisiológicos e bioquímicos das epilepsias ${ }^{14}$. Sendo assim, nesta revisão iremos verificar a utilidade destes estudos em fatias cerebrais na epilepsia do lobo temporal.

\section{EQUIPAMENTO E TÉCNICA}

O tecido cerebral é fatiado por intermédio de um vibrátomo especial com espessura padronizada, dependendo do estudo pretendido. Todo procedimento deve ser realizado com o tecido submerso em solução de Krebs, a qual se assemelha ao lícuido cefalorraquiano. As fatias são mantidas neste meio até

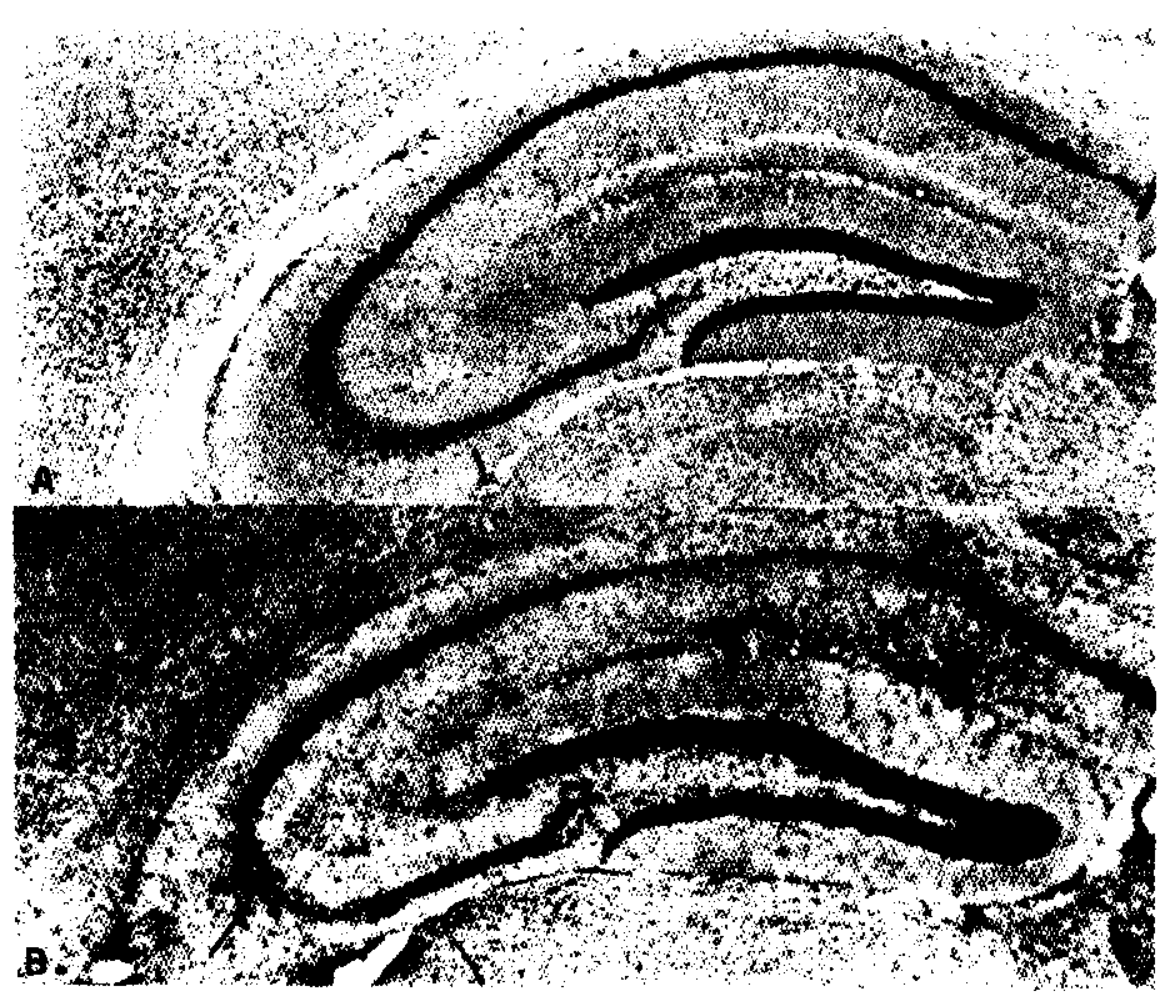

FIGURA 1

Formação hipocampal (coloração de violeta de cresil) A: Citoarquitetura normal de um camundongo pertencente ao grupo-controle. B: Alterações patológicas observadas em um camundongo 162 dias após a administraçāo sistêmica de pilocarpina. Notar a severa perda neuronal nas regiões CA1 e CA3 do hipocampo (setas) (reproduzida com autorização dos autores, Cavalheiro e col., 1996). 
serem transferidas para a câmara de registro eletrofisiológico. Na câmara de registro, as fatias são banhadas com solução de Krebs (líquor cefalorraquiano artificial), em ambiente úmido e oxigenado. Após este procedimento, deixa-se o "slice" descansar por um período de 30 a 60 minutos. Durante este período, as micropipetas de registro e microeletrodos de estimulação são posicionados nos neurônios e vias neurais selecionados para o experimento.

\section{ESTUDOS IN VITRO ("BRAIN SLICES") NA EPILEPSIA DO LOBO TEMPORAL}

\section{Os objetivos principais dos estudos eletrofisiológicos em "slices" cerebrais são:}

- Descrição das propriedades celulares e biofísicas dos neurônios epiléticos;

- Avaliação da função sináptica (fenômenos plásticos) e de sistemas de neurotransmissores;

- Determinação dos eventos patológicos celulares, sinápticos e moleculares (canais iônicos) envolvidos na epileptogênese;

- Determinação de áreas geradoras ("trigger"), marcadoras de ritmicidade ("pacemaker"), assim como mecanismos de propagação e sincronização da atividade epileptiforme.

\section{Técnicas eletrofisiológicas freqüentemente usadas nestes estudos}

- Registros extracelulares (registros de potenciais de campo pós-sinápticos geralmente evocados por estimulação da aferência axonal). Estas estimulações podem ser ortodrômicas (no sentido do fluxo fisiológico dos potenciais de ação) ou antidrômicas (no sentido contrário destes potenciais de ação);

- Registros intracelulares;

- Registros de "patch clamp" em diferentes modalidades ${ }^{76,32}$.

\section{BREVE INTRODUÇÃO DA EPILEPSIA DO LOBO TEMPORAL (ELT)}

A epilepsia do lobo temporal é responsável por um grande número de casos de refratariedade farmacológica a drogas anticonvulsivantes convencionalmente usadas. Estes pacientes apresentam uma sintomatologia clínicat caracterizada por crises parciais complexas que se originam nas estruturas mesiais do lobo temporal, als quais sāo frequientemente de difícil controle. Em muitos casos, a ressecção cirúrgica da formação hipocampal permite um controle das crises ${ }^{49}$.

Embora diferentes estruturas relacionadas com a epileptogênese tenham sido estudadas nos "slices" cerebrais, como neocórtex, córtex piriforme e amídala, focalizaremos esta revisão no hipocampo. O hipocampo tem sido o "protótipo" de estrutura pari a análise da atividade epileptiforme e seus mecanismos "in vitro" um envolvimento especial na fisiopatologia da ELT ${ }^{8 i}$. Referindo-se aos "slices" cerebrais, pequenas peçals de hipocampo denominadas "mini slices" têm a capacidade de gerar atividade epileptiformo semelhante às descargas interictais ${ }^{4^{4}}$. Para abordar este tema, é necessária uma breve descrição das áreals e conexões mais relevantes. assim como dis propriedades intrínsecas dos neurônios hipocampais que favorecem o comportamento anormal das redes neurais presentes no hipocampo.

Os pacientes com ELT apresentam um quadro fisiopatológico em diversas estruturas do sistemi límbico ${ }^{85}$. Fundamentalmente, o hipocampo destes pacientes aparece patologicamente lesado. A história da esclerose hipocampal teve início em 1825, quando Bouchet and Cazauvieilh descreveram macroscopicamente a atrofia hipocampal ${ }^{8}$, Maynert $(1867)^{53}$ e Pfleger $(1880)^{69}$ interpretaram cstats alterações como consequência dos distúrbios metabólicos associados às epilepsias ${ }^{49}$. Sommer (1880) foi o pioneiro em descrever os detalhes histológicos, enfatizando o processo de morte neuronal e atrofial na área CAI do hipocampo ${ }^{87.49}$. Os trabalhos anátomopatológicos de Alzheimer' foram significantes na descrição da lesão após o insulto epilético. O termo esclerose mesial temporal foi introduzido mais tarde com o objetivo de descrever de forma geral as modificaçōes patológicas ${ }^{25}$.

A perda neuronal tem sido estudada em diferentes modelos experimentais de epilepsia do lobo temporal ${ }^{12}$. Existe uma vulnerabilidade neuronal seletiva em diversas áreas cerebrais, destacando-se a região CAI e CA3 do hipocampo (setor de Sommer) e do hilo do giro denteado. A figura I mostra um estudo histológico do hipocampo pelo método de violeta de cresil, podendo-se observar acentuada morte neuronal nas regiōes hipocampais citadas acima. Esta figura está relacionada com o padrão de morte neuronal no 


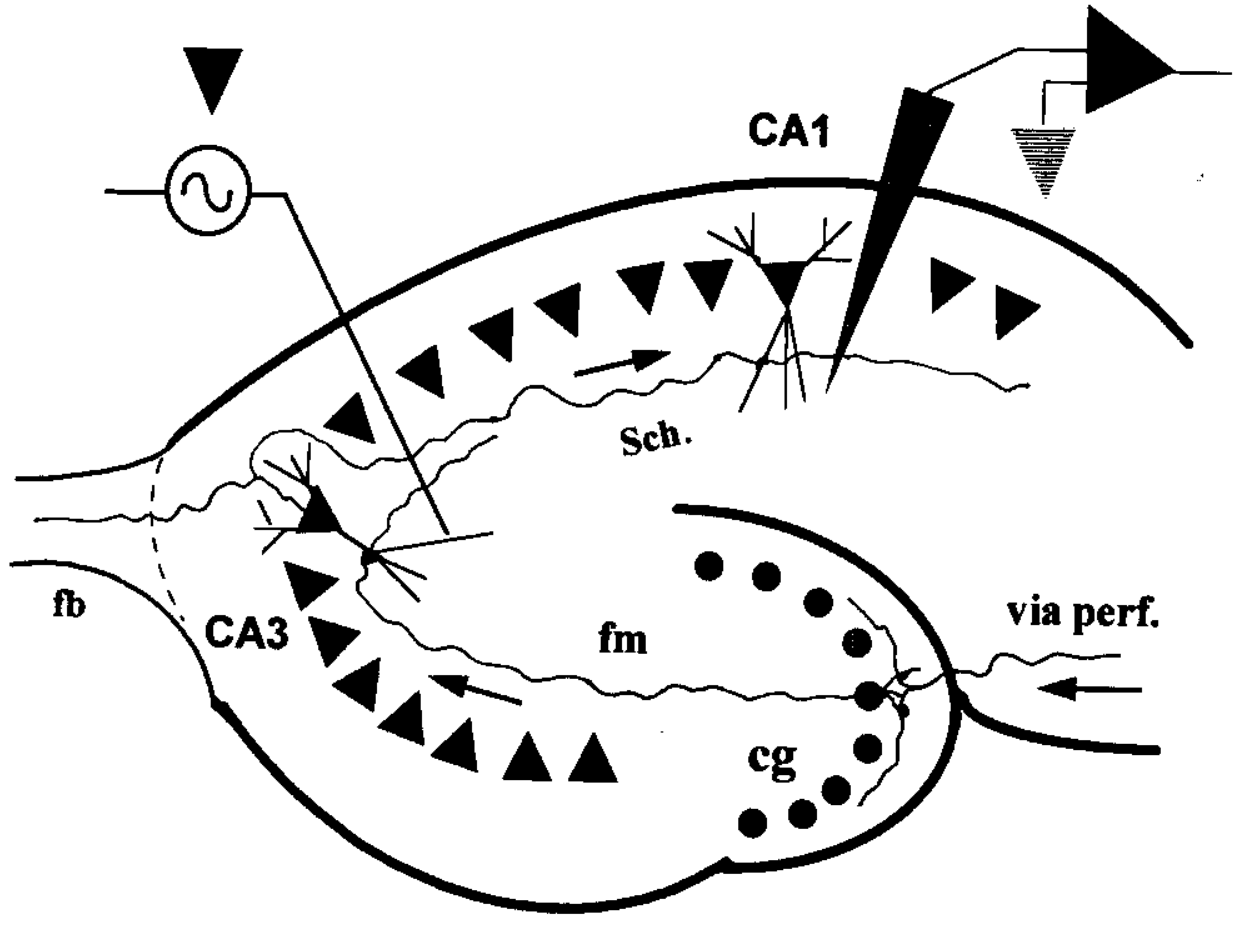

FIGURA 2

Diagrama representativo de um slice de hipocampo mostrando a circuitaria trisináptica e posições de eletrodos de estimulação (área CAl) e registro (área $\mathrm{CA} 3$ ). via perf. via perforante, fm. fibras musgosas, $\mathrm{cg}$. células granulares, fb. fimbria. Sch. via colateral de Schaffer.
Uma correlação entre brotamento de fibras musgosas no giro denteado e alteraçōes eletrofisiológicas tem sido feita em recentes estudos ${ }^{23}$.

\section{PRINCIPAIS COMPONENTES DA FORMACÃO HIPOCAMPAL}

O hipocampo é a parte principal do arquicórtex cerebral. Aparece perto da superfície mesial do lobo temporal e é parcialmente coberto pelo giro parahipocampil. A formação hipocampal engloba quatro regiões: o giro denteado, o corno de Ammon (CA) ou hipocampo propriamente dito, o complexo subicular (parasubiculum, presubiculum e subiculum) e o córtex entorrinal ${ }^{45}$. O corno de Ammon é composto por diferentes áreas de uma camada íntegra de neurônios piramidais denominadas de CA1, CA2, CA3, CA4. As diferentes áreas hipocampais estão ligadas por conexóes axonais hipocampo de camundongos epiléticos crônicos obtidos através do modelo de epilepsia do lobo temporal induzido pela pilocarpina ${ }^{12}$. A figura I A exibe o padrão da coloração de violeta de cresil de um animal-controle em comparação com um animal epilético (figura IB). Os termos esclerose mesial temporal, esclerose do corno de Ammon ou esclerose do hipocampo têm sido freqüentemente usados para caracterizar patologicamente esta entidade. Outra alteração estrutural que acompanha as lesões hipocampais é o brotamento supragranular ("sprouting") das fibras musgosas (formação aberrante de novas ramificações e terminações axonais) das células granulares do giro denteado (figura 3B), sendo que a mesma alteração não é observada nos animais pertencentes ao grupo-controle (figura 3A). Estes fatos têm sido observados em modelos experimentais de epilepsia do lobo temporal como o "kindling" ou "abrasamento", após estimulação elétrica de áreas límbicas específicas descrita por Goddard e colaboradores $(1960)^{13,81}$, no modelo da pilocarpina $^{11.57}$, no modelo de ácido caínico ${ }^{19}$, entre outros. específicas. O giro denteado recebe aferências do córtex entorrinal através da via perfurante (sinapses fundamentalmente excitatórias). Adicionalmente, o córtex entorrinal envia terminaçōes axônicas diretamente para CAl e CA3 (via entorrinohipocampal), sendo as células granulares (CG) o alvo destas conexões. As $\mathrm{CG}$ enviam seus prolongamentos axonais (comumente denominadas de fïbras musgosas) às áreas do hilo do giro denteado e região $\mathrm{CA} 3$, onde fazem sinapse com neurônios piramidais. Dos neurônios piramidais de CA3 emergem axônios (viass colaterais de Schaffer) que terminam em neurônios da região de CAl, efetuando a terceira sinapse do circuito hipocampal. Os neurônios de CAl têm sua região-alvo no subiculum ipsilateral projetando axônios para o córtex entorrinal ipsilateral (terminaçōes sinápticals $n$ à camada IV). Os neurônios subiculares também projetam seus axônios para as camadas do córtex entorrinal e córtex perirrinal ${ }^{45}$, assim como para outrass áreas da formação hipocampal. Resumindo, a vial trisssináptica é formada fundamentalmente por sinapses excitatórias (via perfurante giro denteado CA3 CA1) 
e participa na organização unidirecional do fluxo de informação na formação hipocampal (ver figura 1).

\section{Propriedades intrínsecas dos neurônios hipocampais:}

As propriedades intrínsecas dos neurônios hipocampais são determinadas pela distribuição seletiva de diferentes canais iônicos, como também pelas propriedades intrínsecass da membrana neuronal.

Os íons passam através de canais (poros formados por proteínas) na membrana celular. Estes canais possuem propriedades específicas que garantem sua seletividade iônica, ativação e desativação, assim como sua condutância para cada íon que passa seletivamente por estes canais. A composição eletroquímica dos canais garante o potencial da membrana em repouso, assim como o grau de excitabilidade aos estímulos externos. O fluxo de íns através destes canais gera correntes microscópicas de diferentes intensidades e duração, de acordo com cada canal. Existem normalmente correntes de sódio $\left(\mathrm{I}_{\mathrm{N}}\right)$, de potássio $\left(\mathrm{I}_{\mathrm{K}}\right)$ e diferentes correntes de cálcio $\left(\mathrm{I}_{C_{\mathrm{i}}}\right)$. Estas últimas têm sido bem estudadas na epileptologia devido ao influxo de cálcio para o interior dos neurônios, participando de diversos processos de transdução de sinais, ativação de enzimas e inclusive de fenômenos de transcrição de genes no núcleo das células, o qual pode estar associado aos fenômenos responsáveis pela plasticidade neuronal.

Existem outras correntes importantes no funcionamento eletrográfico destes neurônios: corrente de $\mathrm{K}^{+}$passiva $\left(\mathrm{I}_{\mathrm{L}}\right)$, retificador tardio $\left(\mathrm{I}_{\mathrm{DR}}\right)$, corrente de $\mathrm{K}^{+}$tipo A $\left(\mathrm{I}_{\mathrm{A}}\right)$, corrente de $\mathrm{K}^{+}$de curta duração dependente de voltagem e de cálcio $\left(\mathrm{I}_{\mathrm{Cr}}\right)$, corrente persistente muscarínica $\left(\mathrm{I}_{\mathrm{M}}\right)$ e corrente de $\mathrm{K}^{+}$de longa duraçāo dependente de cálcio $\left(\mathrm{I}_{\mathrm{AH}}\right)$ ou corrente ativada após despolarização.

O canais iônicos podem ser ativados por ligantes, no caso dos receptores glutamatérgicos do tipo NMDA, AMPA, cainato ou metabotrópicos, caracterizando o potencial excitatório sináptico, e também dos receptores GABAérgicos do tipo $\mathrm{GABA}_{\wedge}$ e $\mathrm{GABA}_{13}$ os quais determinam o componente inibitório da resposta sináptica. As correntes iônicas associadas a estes receptores podem ser representadas da seguinte forma: $\mathrm{I}_{\mathrm{NMDN}}, \mathrm{I}_{\mathrm{AMPA}}, \mathrm{I}_{\text {mGl } . U}, \mathrm{I}_{\mathrm{GABA}-\Lambda}, \mathrm{I}_{\mathrm{GABA}-\mathrm{B}}$.

Uma representação resumida da atividade elétrica neuronal é determinada pela corrente absoluta total, que tem sido descrita em diversos modelos biofísicos ou matemáticos baseados nos trabalhos clássicos de
Hodgkin e Huxley (1952). Neste modelo, cada uma dessas correntes seguc uma cinética temporal, dependendo de muitos fatores que podem ser modelados através de equaçōes diferenciais complexals ${ }^{29,93,94}$.

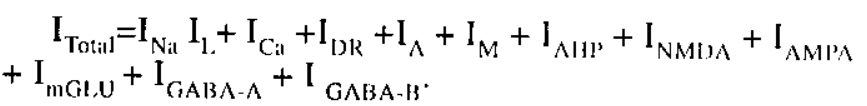

Muitas destas correntes têm sido descritas nas áreas CA 1, CA3 do hipocampo e células granulares do giro denteado ${ }^{13,94,102}$ estando associada aos mecanismos dil gênese da atividade epileptiforme ${ }^{82.102}$. A alteração de diferentes correntes ionicas em modelos de epilepsia crônica ${ }^{15.99}$ sugere que a hiperexcitabilidatde dass redes: neurais do hipocampo é um fenômeno complexo e que talvez seja mediado por uma combinação valriavel das mesmas $^{61.101}$. Em resumo, muitos neurônios hipocatmpais podem apresentar um comportamento anormal que condiciona a suscetibilidade para a geração de crises epiléticas nessas áreas. Essals propriedades elétricals neuronais alteradas pelo processo epilético estão relacionadas com modificaçóes moleculares em relaçĩo aos receptores excitatórios como NMDA. AMPA elc., e canais iônicos da membrana celular. Existem diversias teorias para explicar esse fenômeno, no entanto, a miis aceita seria de um aumento na expressão de canais ionicos ou de receptores com características similares aos normais, como também pelo aumento na expressão de receptores que apresentam uma maior propricdade de excitabilidade. O efeito de diferentes drogas nesses componentes de excitabilidade da membrana pode produzir inibição ou excitação dos mesmos, agindo como uma rede neural epileptogênica, gerando atividade rítmica sincrônica semelhante às descargas epiléticas ${ }^{46,92,94}$.

\section{Participação fisiopatológica da formação hipocampal na gênese da atividade epileptiforme}

Eletrofisiologicamente, podem se encontrar zonas específicas geradoras de descargas epileptogênicals ("trigger"), zonas marcapasos ("pacemaker") ou zonas facilitadoras do ritmo dessas descargas ${ }^{93.94}$. Esse tipo de padrão da atividade tem sido estudado em condições normais e patológicas, como por exemplo, a epilepsia. Alteraçóes na excitabilidade e sincronização dessas redes neurais foram previamente descritas em trabalhos experimentais ${ }^{35.77}$. O estudo das diferentes áreas hipocampais mostram fatos interessantes relacionados às células piramidais da 
região CA3 do hipocampo, como por exemplo, o grande número de canais iônicos ativados por voltagem (ex: canais de cálcio), ou ativados por ligantes (receptores NMDA e AMPA), o quais determinan diferentes graus de excitabilidade, podendo dessa maneira, produzir descargas "explosivas" ("burst") semelhantes aos "paroxismal despolarization shift" (PDS), característicos às descargas neuronais durante a atividade epilética interictal ${ }^{72.83}$. No modelo crônico de ELT induzido por
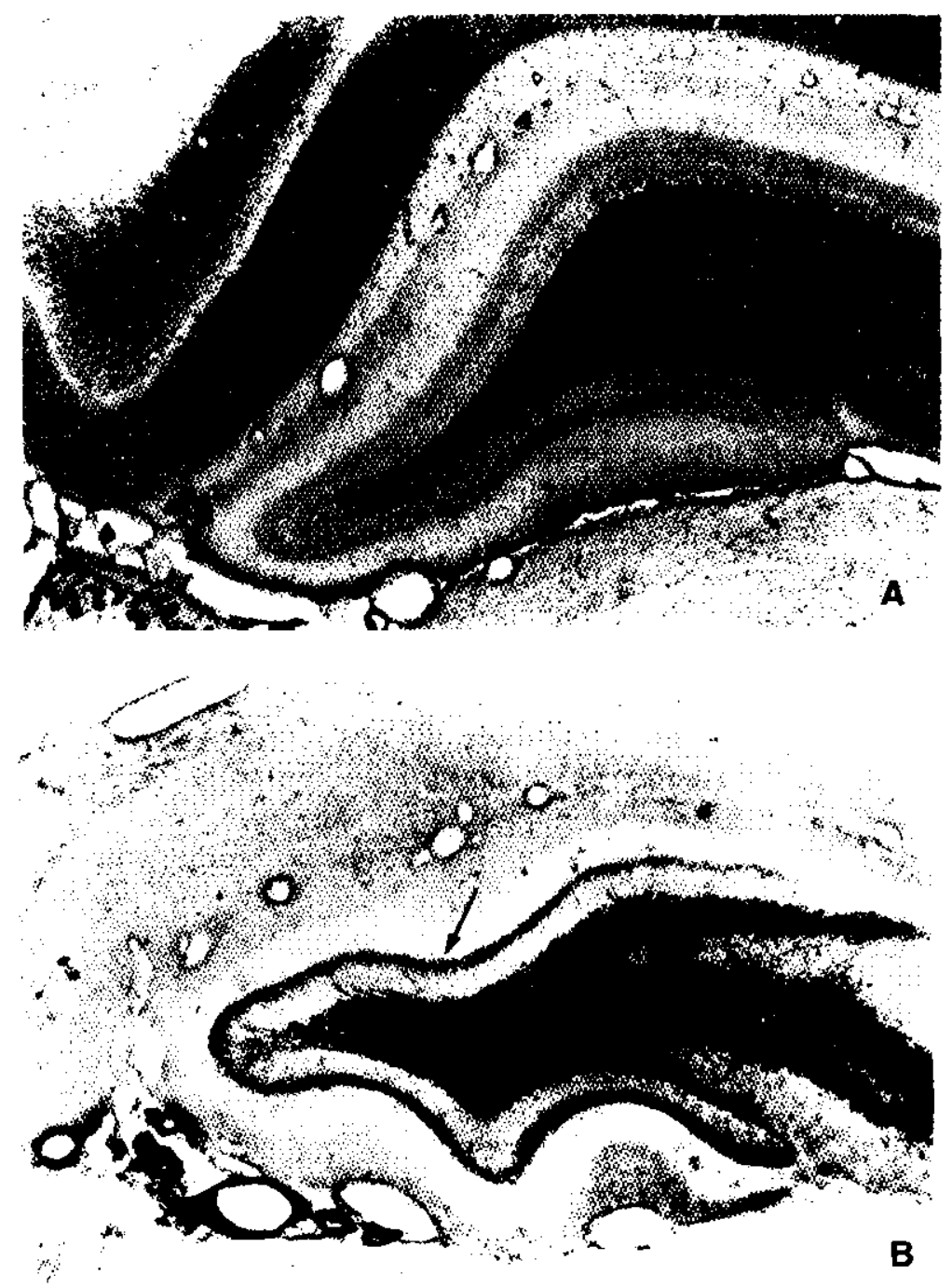

FIGURA 3

Coloração pelo método de Timm da região supragranular da formação hipocampal de ratos. A: Animais pertencentes ao grupocontrole. B: Animais epiléticos, podendo-se notar que os grânulos de Timm estão distribuídos em uma faixa densa e contínua do giro denteado (seta). Notar a atrofia e deformaçāo estrutural do hipocampo. ácido caínico, tem-se investigado a plasticidade sináptica da atividade epileptiforme re-induzida e mediada por receptores NMDA e AMPA $^{7}$. Demonstrou-se uma potenciação a longo prazo da resposta excitatória sináptica mediada por ambos os tipos de receptores glutamatérgicos, podendo-se observar também um aumento na duração das descargas epileptiformes. Interessantemente, estes autores demonstraram que uma estimulação tetânica de alta frequêencia $(100 \mathrm{HZ})$ é capaz de suprimir os eventos sinápticos mediados por NMDA, o que parece ser um mecanismo anticonvulsivante intrínseco no hipocampo. Estas propriedacles elétricas neuronais modificadas pelo processo epilético apresentam a expressão alterada de suas bases moleculares em relação aos receptores excitatórios tipo NMDA ou nāoNMDA e canais iônicos da membrana celular ${ }^{63,64}$. Estudos anteriores sugeriam umi excitabilidade anormal em "slices" de hipocampo de ratos epiléticos submetidos a modelo de "kindling" 35 . Existem diversas teorias para explicar este fenômeno. porém a mais aceita seria de um aumento na expressão de canais jonicos ou de receptores com características similares aos normais, como também pelo aumento na expressĩo de receptores que apresentam uma maior excitabilidade. A novas redes neurais epileptogênicas garantem a manutenção da atividade epileptiforme crônical ${ }^{47.48}$. Além destes latores estruturais, os componentes dals redes neurais do hipocampo mostram uma hiperexcitabilidade atípica quando estudados por intermédio de registros profundos ou a partir de tecido obtido em ciru érgias de pacientes epiléticos de difícil controle farmacológico. Esta última modalidade de estudo eletrofisiológico do tecido "epileptogênico" só é possível através do procedimento de "slices".

Na fisiopatologia da epilepsia, acredita-se que exista um desecpuilíbrio entre mecanismos excitatórios (dependente fundamentalmente de receptores glutamatérgicos) e inibitórios (dependente do GABA) ${ }^{18.55}$. As alterações estruturais nas subunidades desses receptores ou as possíveis mudanças nas propriedades funcionais dos mesmos podem levar a uma condição epileptogênica ${ }^{3,110}$. O estudo eletrofisiológico" in vitro" das redes neurais da formação hipocampal pode auxiliar no esclarecimento das vias neurais e 
sinapses que estão funcionalmente alteradas no hipocampo de pacientes com ELT.

Os achados experimentais sugerem uma alteração dos processos mediados por receptores glutamatérgicos no tecido "epilético" "33,37,38,39,410,51.52,601,61,62,83,96,97. Em "slices" de hipocampo, a atividade epileptiforme tem sido associada com uma função anômala do receptor do glutamato (tipo NMDA) $21.22 .59 .70,92$. Por outro lado, não se descarta a possibilidade da existência de receptores funcionalmente alterados ("epiléticos") que estão sendo patologicamente expressos em neurônios "epiléticos"3.21.22.40,55.56,83,99. Sendo assim, pode-se dizer que existem duas escolas clássicas: a escola do "neurônio epilético" "71.72, a qual defende a existência de populações neuronais intrinsecamente anormais e uma outra que postula a existência de um "agregado epilético"20), ou seja, a atividade epilética é uma propriedade emergente dos circuitos e sinapses funcionalmente alterados ${ }^{83}$.

Uma perspectiva futura está relacionada com o teste farmacológico seletivo de drogas atuantes no tecido epilético. Pacientes resistentes ao tratamento farmacológico clássico podem apresentar receptores alterados ou que sua própria rede neural não responde satisfatoriamente às drogas anticonvulsivantes.

\section{Novos circuitos excitatórios recorrentes associados ao brotamento de fibras musgosas "sprouting" no giro denteado de animais epiléticos crônicos}

O brotamento de fibras musgosas na camada molecular interna do giro denteado tem sido considerado um dos fatos estruturais marcantes na neuropatologia da epilepsia do lobo temporal (figura 3). Muitos pesquisadores têm se interessado na significância fisiológica deste fato. Neste sentido, alguns consideram que o "sprouting" é um mecanismo compensatório não relevante para a epileptogênese crônica. Sloviter em 1994 sugeriu que "sprouting" é fundamentalmente das células inibitórias em cesto ("basket cells"), o que restabeleceria o nível normal de inibição das células granulares ${ }^{86}$. Respostas inibitórias alteradas, dependentes de receptores GABAérgicos, foram estudadas nas células granulares do giro denteado em "slices" de pacientes com esclerose do lobo hipocampal ${ }^{98}$. No entanto, outros autores consideram o "sprouting" um mecanismo aberrante de formação de nova circuitaria excitatória. Tauck and Nedler (1985) foram os primeiros pesquisadores que demonstraram resultados eletrofisiológicos indicando que o brotamento de fibras musgosas poderia formar circuitos excitatórios recorrentes". A partir do modelo do ácido caínico, os mesmos autores verificaram que a estimulação elétricia antidrômica das células granulares do giro denteado pode evocar múltiplas populações de espículas ("population spikes"). Esta resposta poderia ser potenciada por um estímulo condicionante, sendo cjue essas anormalidades estariam relacionadas com a intensidade do "sprouting". Baseando-se em estudos preliminares em relação aos circuitos recorrentes excitatórios em CA3 $3^{15,58}$, Cronin e colaboradores (1992) elaboraram a hipótese de que os novos circuitos excitatórios recorrentes entre as células granulares são mascarados por uma inibição recorrente ${ }^{17}$. Estudos em "slices" de hipocampo, sob condições de inibição reduzida, estes autores supoem que o estímulo elétrico na região do hilo pode evocar respostas com atividade epileptiforme nas células granulares ${ }^{17}$ Outros autores têm associado resultados eletrofisiológicos às características das células granulares presentes no hipocampo de animais epiléticos crônicos induzidos pela pilocarpina $^{31}$ como também em tecido obtido em cirurgia de pacientes epiléticos ${ }^{30}$. Pesquisas recentes no modelo do ácido caínico indicam que a suscetibilidade às crises recorrentes em animais que apresentam brotamento das fibras musgosas, deve ser dependente da formação de novas sinapses excitatórias ${ }^{1(4)}$. É muito provável que o grau de excitabilidade da nova rede neural seja determinado pelo desbalanço entre os eventos excitatórios e inibitórios ${ }^{88}$. Essa reorganização sináptica aberrante acontece no transcorrer do tempo após um insulto, como por exemplo, o status epilepticus ${ }^{57,85.86 .88 .8 \%}$ Em estudos recentes têm-se estudado o brotamento de fibras musgosas no giro denteado em preparações e cultivos (in vitro) de "slices" de hippocampo ${ }^{18}$.

\section{ESTUDOS EM ANIMAIS}

\section{Modelos de epilepsia desenvolvidos "in vitro" a partir de "slices" de tecido cerebral}

Diferentes estruturas cerebrais têm sido estudadas nestes modelos. As mais estudadas são "slices" de hipocampo e "slices" combinados de córtex entorrinal e hipocampo, preservando-se a integridade da via trissináptica. 
As classificações desses modelos "in vitro" de atividade epileptiforme baseiam-se em formas existentes de geração da atividade epilética.

\section{Modelos induzidos farmacologicamente:}

A Especialmente por bloqueio seletivo de receptores inibitórios GABAérgicos do tipo A mediante diversas drogas (ex. bicuculina, picrotoxina ou penicilina) 20137.

$B$ Aplicação de drogas ativadoras de receptores glutamatérgicos. O NMDA tem sido usado para produzir atividade epileptiforme "in vitro" 2.21 .

C Bloqueio do canal iônico de potássio (responsável pela hiperpolarização fisiológica da membrana neuronal). Uma droga amplamente usada é a 4-aminopiridina (4AP). A 4-AP aumenta a excitabilidade neuronal intrínseca, aumentando e prolongando os potenciais sinápticos ${ }^{75.106}$. O aumento da concentração de potássio também tem sido usado para produzir atividade epileptiforme in vitro ${ }^{15}$.

\section{Modelos Induzidos por manipulações iônicas e metabólicas do meio:}

Perfusão do meio que banha o tecido na ausência de ions de magnésio $\left(\mathrm{Mg}^{2+}\right)$ (epilepsia por baixo teor de $\left.\mathrm{Mg}^{2+}\right)^{62,(1)} . \mathrm{O} \mathrm{Mg}^{2+}$ produz um bloqueio dependente de voltagem no complexo canal iônico/receptor de NMDA ${ }^{92}$. Porém, a falta deste íon condiciona um estado de hiperexcitabilidade neuronal pelo aumento da condutância destes receptores, aumentando a liberação sináptica de neurotransmissores ${ }^{41.61 .63}$.

\section{Estudo "in vitro" do tecido cerebral em modelos de epilepsia induzidos "in vivo"}

Estes estudos apresentam algumas particularidades, destacando-se a reindução da atividade epileptiforme ou o funcionamento e plasticidade sináptica dos circuitos neurais (potenciação a longo prazo $=$ long-term potentiation).

No caso da reindução da atividade epileptiforme, destacam-se os trabalhos de Nagao e colaboradores em $1994^{6.5}$. Nesta investigação, a 4-AP é aplicada em "slices" de hipocampo para a análise do padrão eletrofisiológico, após a indução de descargas epileptiformes no hipocampo de ratos que apresentam crises espontâneas e recorrentes, em comparação com "slices" de ratos normais. As propriedades de excitabilidade mediada por NMDA, têm sido estudadas neste mesmo modelo ${ }^{31}$, sendo relacionadas às alterações estruturais dos neurônios analisados. Recentemente, alguns autores registraram um aumento na duração da resposta sináptica mediada por glutamato (potenciais excitatórios pós-sinápticos, PEPs) nas células granulares do giro denteado de ratos. Este mesmo grupo de pescuisadores observam modificações semelhantes presentes no hipocampo de pacientes epiléticos ${ }^{30}$.

\section{Estudos da plasticidade sináptica hipocampal do tecido epilético}

As mudanças funcionais e estruturais após um insulto, como o status epilepticus, parece ser um processo lento e progressivo, o qual leval a uma condiçäio crônica. Esses fenômenos de epileptogênese têm sido comparados ao processo de potenciação de longa duração da função sináptica (LTP), processo asssociado com um aumento na função dependente de receptores glutamatérgicos ${ }^{5.6 .16}$

Os estudos de plasticidade sináptica no tecido epilético são bem variáveis. Em prévios estudos demonstrou-se que a atividade epileptiforme induzidal "in vitro" é capaz de alterar os processos sinápticos de potenciação de longa duração da atividade sináptica ${ }^{3,5,6,1(1)}$. Esta forma de plasticidade sináptical dependente de estímulos, está sendo associada aos mecanismos celulares e moleculares responsáveis pela formação e consolidação da memória ("in vive"), porém, uma alteração da mesma pode explicalr a fisiopatologia da amnésia, freqüentemente observalda em pacientes epiléticos. Recentes estudos sugerem cjue os mecanismos epileptogênicos podem produzir mudanças semelhantes ao processo de plasticidade ${ }^{+2.43}$. Tem-se demonstrado que a atividade epileptiforme pode produzir alterações na potenciação e depressão da função sináptica a longo prazo ${ }^{16}$. Estudos recentes em "slices" de hipocampo no modelo crônico de ELT, sugerem hiper-respostas excitatórias na região CAI do hipocampo e giro denteado após a estimulação aferente ${ }^{74}$. Estes resultados estão de acordo com estudos anteriores em tecido cerebral de pacientes epiléticos crônicos submetidos à cirurgia para extração do foco epilético ${ }^{83.84}$. Adicionalmente, foram encontradas alterações na neurotransmissão GABAérgica induzida após a estimulação das mesmas áreas da formação hipocampal (CAl e giro denteado) neste modelo de ELT ${ }^{47.48 .50}$, como também no "kindling"63.67. As alteraçōes descritas na expressão e funcionamento de receptores AMPA e NMDA no 
processo de epileptogênese ${ }^{66}$ também contribuem na alteração da fisiologia das sinapses. Vários autores correlacionam as alterações plásticas "in vitro" aos distúrbios na função dos receptores $\mathrm{GABA}_{\wedge}{ }^{78}$. Esses resultados estão de acordo com estudos eletrolisiológicos previamente realizados em pacientes epiléticos ${ }^{98.103}$

O fenomeno de plasticidade é muito interessante, pois a potenciação sináptica em determinadas vias facilita o processo de "kindling"18.65. Um dos primeiros estudos mostrando este fenomeno de plasticidade foi realizado na via perfurante ${ }^{88}$. Outro fato amplamente estudado em "slices" de hipocampo é a alteração na expressão de canais iônicos, como também as alterações existentes nas propriedades fisiológicas intrínsecals da membrana neuronal após a manutençāo da atividade epilética ${ }^{59,70,97,(1)}$. Em resumo, pode-se dizer que o processo de epileptogênese está relacionado com fenômenos plásticos induzidos pela atividade epilétical 9.19 .63 .65 .78 .

\section{ESTUDOS EM HUMANOS}

\section{Estudos eletrofisiológicos "in vitro" em "slices" de hipocampo humano obtidos de pacientes submetidos à cirurgia para o tratamento da epilepsia}

As primeiras investigaçoes eletrofisiológicas em tecido humano datam de $1955^{101}$. Tem-se demonstrado, posteriormente, que as células neocorticais e hipocampais de pacientes com ELT apresentam propriedades eletrofisiológicas semelhantes àquelas observadas em modelos experimentais ${ }^{79.81 .83 .1617 .1118}$

Os primeiros estudos "in vitro" com tecido humano foram possíveis após a adaptação da técnica de registro intracelular em "slices" desenvolvida por Yamamoto e Schwartzkroin na década de $1970^{79.82 .1(17.108}$. O primeiro registro extracelular no tecido humano "in vitro" foi realizado em 1973 por Kato e colaboradores ${ }^{34}$. Já o registro intracelular em neurônios do tecido humano foi relatado em 1976 por Schwartzkroin e Prince ${ }^{49}$. Estes estudos levaram ao rápido desenvolvimento destes procedimentos em regiões neocorticais e estruturas mesiais temporais como o hipocampo, amídala e córtex entorrinal ${ }^{49,71,72}$.

Embora estas propriedades tenham certa variabilidade, foi determinado, por meio da análise quantitativa de 111 neurônios neocorticais do lobo frontal e temporal, a medida do potencial de membrana destes neuronios que foi de aproximadamente $-6.5 \mathrm{mV}$, a amplitude do potencial de ação foi de aproximadamente $85 \mathrm{mV}$ e a resistência elétrica de 40 ohms ${ }^{4}$. Outros tipos de correntes também foram avaliadals neste estudo, como a corrente de $\mathrm{K}^{+}$dependente de $\mathrm{Ca}^{2+}\left(\mathrm{I}_{\mathrm{NuH}}\right)$, que participa dos fenômenos subjacentes à atividade interictal. Posteriormente, estas correntes ionicas foram analisadas através da técnica eletrofisiológica de "voltage clamp" 28 .

Muitos trabalhos foram realizados com intuito de elucidar os mecanismos básicos que sustentam a hiperexcitabilidade dos neurônios epiléticos humanos ${ }^{79,80,82}$. Umas das hipóteses mais interessantes está relacionada com uma alteração dos potenciais inibitórios GABAérgicos. Por outro lado, supõe-se que certas células neocorticais poderiam induzir descargas tipo "burst" após a estimulaçĩo, geradas possivelmente por mecanismos sinápticos ${ }^{83.84}$. Com $o$ intuito de diferenciar as propriedades eletrofisiológicas dos neurônios de alta e de baixa epileptogenicidade, trabalhos realizados anteriormente não detectaram descaryas epileptiformes espontâneas do tipo PDS. Os neurônios de alta epileptogenicidade apresentaram um aumento gradativo da resposta sináptica evocada por estímulos ${ }^{4}$. Outros autores sugerem que os potenciais de ação evocados por estimulação devem atuar como marcadores de epileptogenicidade em neurônios humanos ${ }^{49}$, fato que poderia corrresponder ao baixu limiar da descarga elétrica neuronal. Até o momento, nāo tem sido demonstrada uma atividade de descargas epileptiformes espontâneas no tecido epilético humano, o qual tem sido observado em estudos de eletrocorticograma intra-operatório "in situ” (ECoG). Em 1989, Reid e Palovcik relatam a presença de potenciais de campo espontâneos e sincrônicos parecidos com descargas interictais em "slices" de tecido neocortical, obtido de zonas com descargas eletrográficas previamente detetadas por meio do ECoG ${ }^{13}$. Esta atividade sincrônica "in vitro" não tem sido encontrada nas zonas inativas ou "silentes". Uma das dificuldades clássicas e não bem-resolvida na atualidade é a inexistência de tecidos-controle nestes estudos. Têm-se empregado diferentes métodos para uma análise comparativa, utilizando-se tecido de primatas e tecido obtido em cirurgias para remoção de tumores cercbrais, porém os detalhes éticos e metodológicos são muito complexos nestes casos. Uma possibilidade mais objetiva foi sugerida por Liiders (1992), na qual comparou os diferentes graus de epileptogenicidade no tecido humano em relação às alterações patológicas existentes ${ }^{49}$. Estas 
investigações foram realizadas em tecido epilético de crianças submetidas a ressecção cirúrgica ${ }^{23,90}$.

Alterações do componente NMDA na resposta sináptica têm sido encontradas em tecido hipocampal ("slices") obtido em cirurgias de pacientes epiléticos ${ }^{51.52 .97}$. Esse mesmo grupo de pesquisadores têm correlacionado as alterações estruturais na circuitaria hipocampal (ex.: grau de brotamento de fibras musgosas) aos distúrbios eletrofisiológicos ${ }^{17.311 .911 .91 .96 .97}$. O receptor do tipo AMPA parece também estar afetado na patologia epilética humana analisada "in vitro" $51,52,55,56,73$. Por outro lado, diversos trabalhos realizados com tecido obtido de pacientes epiléticos têm enfatizado as propriedades eletrofisiológicas das conexões e sinapses do hipocampo responsáveis pela hiperexcitabilidade ${ }^{36.83 .84,90}$.

Em contrapartida, estudos farmacólogicos em "slices" de hipocampo "in vitro" estão sendo realizados com o intuito de se avaliar a ação de drogas anticonvulsivantes ${ }^{2,66,110}$. Isso possibilita maiores informações sobre o mecanismo de ação das drogas anticonvulsivantes.

Em resumo, várias questões devem ser esclarecidas com relação à gênese da atividade epileptiforme no tecido "epilético": 1) Existe uma redução da inibição no tecido "epilético"? 2) As células do tecido epilético são mais suscetíveis a descargas epileptiformes em resposta à estimulação? 3) Existe uma contribuição exagerada e patológica dos receptores excitatórios glutamatérgicos nos potenciais sinápticos ou descargas epileptiformes? 4) As mudanças estruturais como "sprouting" estão correlacionadas com o aumento da excitabilidade?

\section{SUMMARY}

Temporal lobe epilepsy is a common neurological disorder and, perhaps, a window into the nature of neuronal network defects that result in pathological hyperexcitability. Mesial temporal structures play an important and probably predominant role in the causation of complex partial seizures encountered in patients with temporal lobe epilepsy. This evidence has represented the rationale for numerous studies in vivo and in vitro in models of epilepsy in which the main emphasis has been directed toward the physiopathology of the hippocampus proper. Experiments pertormed in the in vitro hippocampal slice preparation have revealed several fundamental cellular and pharmacological mechanisms responsible for the generation of epileptiform discharges. The brain stice techinique olfers the advantages of physical stability, which allows intracellular recording, control over the extracellular ionic medium and $\mathrm{pH}$, and drug aplication. The brain tissue is, of course, isolated from other neural and hormonal inputs, which can be considered an advantage and a disadvantage of the method.

Eletrophysiological studies of brain slices have been centered around some main objectives: 1) descriptions of the cellular biophysical and synaptic properties of neurons, 2) investigations of neurotransmitter function in the brain. that an important issues in determining cellular and synaptic pathologies causing epileptogenesis.

\section{KEYWORDS}

Brain "slices", blectrophysiology, temporal lobe epilepsy, hippocampal sclerosis.

\section{Referências}

1. Alzheimer, A. Ein beitrag zur pathologischen anatomie der epilepsie. Mschr. Neurol, 4: 345-369, 1898.

2. Apland, J.P.; Cann, F.J. Anticonvulsant effects of memantine and MK-801 in guinea pig hippocampal slices. Brain Res Bull, 37: 311-316, 1995.

3. Ashwood, T.J.; Wheal, H.V. The expression of N-methyl-Daspartate receptors-mediated component during epileptiform synaptic ectivity in hippocampus. Br. J. Pharmacol., 91: 815822, 1987.

4. Avoli, M.; Olivier, A. Bursting in human epileptogenic neocortex is depressed by an $\mathrm{N}$-methyl-D-aspartate antagonist. Neurosci. Lett., 76: 249-254, 1987.

5. Bashir, Z.I.; Alford, S.; Davies, S.N.; Randall, A.D.; Collingridge, G.L. Long-term potentiation of NMDA receptormediated synaptic transmission in the hippocampus. Nature, 349: 156-158, 1991.

6. Baudry, M. Long-term potentiation and kindling: Similar biochemical mechanisms? Advances in Neurology. Vol 44. ed. A.V. Deigado-Escueta. A.A Ward Jr.. D.M. Woodbury. \& R.J., Porter. Raven Press. New York.. 401-407, 1986.

7. BERNARD, C. \& WHEAL, H. - Plasticity of AMPA and NMDA receptor-mediated epileptiform activity in a chronic model of temporal lobe epilepsy. Epilepsy Res., 21: 95-107, 1995.

8. Bouchet, C.; Cazauvieilh De l'epilepsie consideree dans ses rapports avec la l'alienation mentale. Arch Gen Med., 9: 1042, 1825.

9. Cain, D.P. Long-term potentiation and kindling: how similar are the mechanisms?. Trends Neurosci.,12: 6-10, 1989.

10. Cain, D.P.; Hargreaves, E.L.; Boon, F.; Dennison, Z. An examination of the relation between hippocampal long-term potentiation, kindling, afterdischarge, and place learning in the water maze. Hippocampus, 3: 153-164, 1993.

11. Cavalheiro, E.A.; Leite, J.P.; Bortolotto, Z.A.; Turski, W.A.; Ikonomidou, C.; Turski, L. Long-term effects of pilocarpine in rats: Structural damage of the brain triggers kindling and spontaneous recurrent seizures. Epilepsia, 32: 778-782, 1991.

12. Cavalheiro, E.A.; Santos; N.F.; Priel, M.R. The pilocarpine model of epilepsy in mice. Epilepsia, 37: 1015-1019, 1996.

13. Cavazos, J.E.; Golarai, G.; Sutula, T.P. Mossy fiber synaptic reorganization induced by kindling: Time course of development. progression and permanence. J. Neurosci., 11: 2795- 2803, 1991

14. Collingridge, G.L. The brain slice preparation: a tribute to the pioneer Henry Mcllwain. J. Neurosci., 59: 5-9, 1995.

15. Colom, L.V.; Saggau, P. Spontaneous interictal-like activity originates in multiple areas of the CA2-CA3 region of hippocampal slices. J. Neurophysiol., 71: 1574-1585, 1994.

16. Contzen, R.; Witte, O.W. Epileptic activity can induce both long-lasting potentiation and long-lasting depression., Brain Res., 653: 340-344, 1994.

17. Cronin, J.; Obenaus, A.; Houser, C.R.; Dudek, F.E. Electrophysiology of dentate granule celts after kainateinduced synaptic reorganization of the mossy fibers. Brain Res., 573: 305-310, 1992.

18. Dailey, M.E.; Buchnan, J.; Bergles, D.E.; Smith, S.J. Mossy fiber growth and synaptogenesis in rat hippocampal slices in vitro. J. Neurosci., 14: 1060-1078, 1994.

19. Davenport, C.J.; Brown, W.J.; Babb, T.L. Sprouting of GABAergic and mossy fiber axons in dentate gyrus following intrahippocampal kainate in the rat. Exp. Neurol., 109: 180. 190,1990

20. Dichter, M.; Spencer, W.A. Penicillin-induced interictal discharges from the cat hippocampus. II. Mechanisms underlying origin and restriction. J. Neurophysiol., 32: 663$687,1969$. 
21. Dingledine, R.; Hynes, M.A.; King, G.L. Involvement of Nmethyl-D-aspartate receptors in epileptifoerm bursting in the rat hippocampal slice. J. Physiol., 380: 175-189, 1986.

22. Dingledine, R.; McBain, C.J.; McNamara, J.O. Excitatory amino acid receptors in epilepsy. Trend in Pharmacol. Sci., 11: 334-338, 1990.

23. Dudek, F.E.; Obenaus, A.; Schweitzer, J.S.; Wuarin, J.P. Functional significance of hippocampal plasticity in epileptic brain: Electrophysiological changes of the dentate granule cells associated with mossy fiber sprouting. Hippocampus, 4: 259-265, 1994

24. Edwards, F.A.; Konnerth, A.; Sakmann, B.; Takahashi, T. A thin slice preparation for patch-clamp recordings from neurones of the mammalian central nervous system. Instruments and techniques. Pflügers Arch., 414: 600-612, 1989.

25. Gastaut, H. So called "psychomotor" and "temporal" epilepsy. A critical study. Epilepsia, 2: 57-70, 1953.

26. Gibson, I.M.; Mcllwain, H. Continuous recording of changes in membrane potential in mammalian cerebral tissues in vitro; recovery after depolarization by added substances, J. Physiol., 176: 261-283, 1965.

27. Goddard, G.V.; McIntyre, D.C.; Leech, C.K. A permanent change in brain function resulting from daily electrical stimulation. Expl. Neurol., 25: 295-330, 1960.

28. Halliwell, J.V. Cholinergic responses in human neocortical neurones. Experientia Suppl., 57: 138-49, 1989.

29. Hodgkin, A.L.; Huxley, A.F. A quantitative description of membrane current and its application to conduction and excitation in nerve. J. Physiol., 117: 500-544, 1952.

30. Isokawa, M.; Levesque, M.F. Increased NMDA responses and dendritic degeneration in human epileptichippocampal neurons in slices. Neurosci. Lett., 132: 212, 1991.

31. Isokawa, M.; Mello, L.E. NMDA receptor-mediated excitability in dendritically deformed dentate granule cells in pilocarpine treated rats. Neurosci. Lett., 129: 69-73, 1991.

32. Jonas, P.; Sakmann, B. Glutamate receptor channels in isolated patches from $\mathrm{CA} 1$ and CA3 pyramidal cells of rat hippocampal slices. J. Physiol., 455: 143-171, 1992

33. Kamphuis, W.; Lopes da Silva, F.H.; Wadman, W.J. Changes in evoked potentials in the rat hippocampus (CA1) during kindling epileptogenesis. Brain Res., 440: 205-215, 1988.

34. Kato, H.; Ito, Z.; Matsuoka, S.; Sakurai, Y. Electrical activities of neurons in the sliced human cortex in vitro. Electroencephalogr. Clin. Neurophysiol., 35: 457-462, 1973.

35. King, G.L.; Dingledine, R.; Giacchino, J.L.; McNamara, J.O. Abnormal neuronal excitability in hippocampal slices from kindled rats. J. Neurophysiol., 54: 1295-1304, 1985.

36. Knowles, W.D.; Awad, I.A.; Naye, L.M. Differences of in vitro electrophysiology of hippocampal neurons from epileptic patients with mesiotemporal sclerosis versus structural lesions. Epilepsia, 33: 601-609, 1992.

37. Körh, G.; Heinemann, U. Effects of NMDA antagonists on picrotoxin-, low $\mathrm{Mg}^{2+}$-and $\mathrm{Ca}^{2+}$-induced epileptogenesis and evoked changes in extracellular $\mathrm{Na}^{+}$and $\mathrm{Ca}^{2+}$ concentrations in rat hippocampal slices. Epilepsy Res., 4: 187-200, 1989

38. Körh, G.; Heinemann, U. A long-lasting decrease in the inhibitory effect of GABA on glutamate responses of hippocampal pyramidal neurons induced by kindling epileptogenesis. Neuroscience, 41: 425-431, 1989.

39. Körh, G.; De Koninck, Y.; Mody, I. Properties of NMDA receptor channels in neurons acutely isolated from epileptic (kindled) rats. J. Neurosci., 13: 3612-3627, 1993.

40. Kraus, J.E.; Yeh, G.C.; Bonhaus, D.W.; Nadler, J.V.; McNamara, J.O. Kindling induces the long-lasting expression of a novel population of NMDA receptors in hippocampal region CA3. J. Neurosci., 14: 4196-4205, 1994.
41. Leschinger, A.; Stabel, J.; Igelmund, P.; Heinemann, U. Pharmacological and electrographic properties of epileptiform activity induced by elecated $\mathrm{K}^{+}$and lowered $\mathrm{CA}^{2+}$ and $\mathrm{Mg}^{2+}$ concentration in rat hippocampal slices. Exp. Brain Res., 96: 230-240, 1993.

42. Leung, L.S. Evaluation of the hypothesis that interictal spikes are caused by long-term potentiation. Epilepsia, 35: 785-794. 1994.

43. Leung, L.S.; Zhao, D.; Shen, B. Long-lasting effects of partial hippocampal kindling on hippocampal physiology and function. Hippocampus, 4: 696-704, 1994.

44. Li, C.L.; Mcllwain, H. Maintenance of resting membrane potentials in slices of mammalian cerebral cortex and other tissues in vitro. J. Physiol., 139: 178-190, 1957.

45. Lopes da Silva, F.H. Anatomic and physiologic of limbic cortex., Ann. Rev. Physiol., 1990.

46. Lopes da Silva, F.H.; Kamphuis, W.; Wadman, W.J. Future research in epileptology: Epileptogenesis as a plastic phenomenon of the brain, a short review. Acta Neurol. Scand. Suppl. 140., 86: 34-40, 1992.

47. Lothman, E.W.; Rempe, D.A.; Mangan, P.S. Changes in excitatory neurotransmission in the CA1 region and dentate gyrus in a chronic model of temporal tobe epilepsy., J. Neurophysiology., 74: 841-848, 1995

48. Lothman, EW.; Betrtran, EH.; Kapur, J.; Stringes, J.L. Recurrent spontaneous hippocampal seizures in the rat as chronic sequela to limbic status epilepticus. Epilepsy Res. 6: $110-118,1990$.

49. Lüders, H.O. (ed.) Epilepsy Surgery., New York: Raven Press. 1992.

50. Mangan, P.S.; Rempe, D.A.; Lothman, E.W. Changes in inhibitory neurotransmission in the CA1 region and dentate gyrus in a chronic model of temporal lobe epilepsy. J. Neurophysiol., 74: 829-840, 1995.

51. Masukawa, L.M.; Higashima, M.; Hart, G.L.; Spencer, D.D.; O'Connor, M.J. NMDA receptor activation during epileptiform responses in the dentate gyrus of epileptic patients. Brain Res, 562: 176-180, 1991

52. Masukawa, L.M.; Higashima, M.; Kim, J.H.; Spencer, D.D. Epileptiform discharges evoked in hippocampal brain slices from epileptic patients. Brain Res., 493: 168-174, 1989

53. Mayner, T. Studian über das pathologish-anatomishe material der wiener irrenanstalt. Vierteljahressh Psychiatr., 3: 381-402, 1867.

54. Mcllwain, H.; Buchel, L.; Cheshire, J.D. The inorganic phosphate and phosphocreatine of brain especially during metabolism in vitro. Bioch., J. 48: 12-20, 1951.

55. McNamara, J.O. Cellular and molecular basis of epilepsy. J. Neurosci., 14: 3413-3425, 1994

56. McNamara, J.O. Increased AMPA-sensitive quisquilate receptor binding in epileptic human hippocampus. J. Neurosc., 11: 428-434, 1991

57. Mello, L.E.A.M.; Cavalheiro, E.A.; Tan, A.M.; Kupper, W.R. Pretorius, J.K.; Babb, T.L.; Finch, D.M. Circuit mechanisms of seizures in the pilocarpine model of chronic epilepsy: Cell loss and mossy fiber sprouting. Epilepsia, 39: 985-995, 1993.

58. Miles, R.; Womg, R.K.S. Excitatory synaptic interactions between CA3 neurones in the guinea-pig hippocampus. $J$. Physiol., 353: 397-418, 1986.

59. Mitsuyoshi, I.; Ito, M.; Shirasaka, Y.; Mikawa, H.; Serikawa, T.; Yamada, I. Changes of NMDA receptor binding in spontaneously epileptic rat and parent strains. Neurochem. Res., 18: 1169-1173, 1993

60. Mody, I.; Heinemann, U. NMDA receptors of dentate gyrus granule cells participate in synaptic transmission following kindling. Nature, 326: 701-704, 1987

61. Mody, 1.; Staley, K. Cell properties in the epileptic hippocampus, Hippocampus, 4: 275-280, 1994. 
62. Mody, 1.; Lambert, J.D.C.; Heinemann, U. Low extracellular magnesium induces epileptiform activity and spreading depression in rat hippocampal slices. J. Neurophysiol., 57: 869-888, 1987

63. Mody, I.; Stanton, P.K.; Heinemann, U. - Activation of Nmethyl-D aspartate receptors parallels changes in cellular and synaptic properties of dentate gyrus granule cells after kindling. J Neurophysiol., 50: 1033-1054. 1988

64. Morrisett, R.A.; Chow, C.; Nadier, J.V.; McNamara, J.O. Biochemical evidence for enhanced sensitivity to $\mathrm{N}$-methylD-aspartate in the hippocampal formation of kindled rats. Brain Res., 496: 25-28, 1989

65. Nagao, T.; Avoli, M.; Gloor, P. Interictal discharges in the hippocampus of rats with long-term pilocarpine seizures., Neurosci. Lett., 174: 160-164, 1994.

66. Namba, T.; Morimoto, K.; Sato, K.; Yamada, N.; Kuroda, S. Antiepileptogenic and anticonvulsant effects of NBQX, a selective AMPA receptor antagonist, in the rat kindling model of epilepsy. Brain Res., 638: 36-44, 1994.

67. Oliver, M.W.; Miller, J.J. Inhibitory processes of hippocampal CA1 pyramidal neurons following kindling-induced epilepsy in the rat. Can. J. Physiol. Pharmacol., 63: 872-878, 1985

68. Pelimar, T.C. Use of brain slices in the study of free-radical actions. J. Neurosci. Methods., 59: 85-92., 1995.

69. Pfleger, L. Beobachtungen über schrumptung und sklerose des ammonshorns bei epilepsie. Allgemeine Z. Psychiatr., 36: 359-365, 1880

70. Pratt, G.D.; Kokaia, M.; Bengzon, J.; Kokaia, Z.; Fritschy, J.M.; Möhler, H.; Lindvall, O. Differential regulation of $\mathrm{N}$ methyl-D-aspartate receptor subunit messenger RNAs in kindling-induced epileptogenesis. Neuroscience., 57: 307 . $318,1993$.

71. Prince, D.A.; Wong, R.K.S. Human epileptic neurons studied in vitro. Brain Res., 210: 323-333, 1981.

72. Prince, D.A. Neurophysiology of epilepsy. Ann Rev Neurosci., 1: 395-415, 1978

73. Reid, S.A.; Palovcik, R.A. Spontaneous epileptiform discharges in isolated human cortical slices from epileptic patients. Neurosci. Lett., 98: 200-204. 1989.

74. Rempe, D.A.; Mangan, P.S.; Lothman, E.W. Regional heterogeneity of pathophysiological alterations in CA1 and dentate gyrus in a chronic model of temporal lobe epilepsy. J. Neurophysiol., 74: 816-828, 1995.

75. Retecki, P.A.; Lebeda, F.J.; Johnston, D. 4-Aminopyridine produces epileptiform activity in hippocampus and enhances synaptic excitation and inhibition. J. Neurophysiol., 57: 1911. 1924. 1987.

76. Sakmann, B.; Edwards, F.; Konnerth, A.; Takashi, T. Patchclamp techniques used for studying synaptic transmission in slices of mammaliam brain. Quart. J. Experim. Physiol., 74: 1107-1118, 1989

77. Schafman, H.E. Synchronization of area CA3 hippocampal pyramidal cells and non-granule cells of the dentate gyrus in bicuculline treated rat hippocampal slices. Neurosci. , 59:245. 257, 1994.

78. Schneiderman, J.H.; Sterling, C.A.; Luo, R. Hippocampal plasticity following epileptiform bursting produced by $\mathrm{GABA}_{A}$ antagonists. Neuroscience, 59: 259-273, 1994.

79. Schwartzkroin, P.A.; Frank, J.E. Electrophysiology of epileptic tissue: What pathologies are epileptogenic? Adv Exp Med Biol, 203: 157-172, 1986.

80. Schwartzkroin, P.A.; Knowles, W.D. Intracellular study of human epileptic cortex: in vitro maintenance of epileptiform activity? Science, 223: 709-712, 1984.

81. Schwartzkroin, P.A. Basic research in the setting of an epilepsy surgery center. In: surgical treatment of the epilepsies (Engel J, Jr, ed) 755-773, New York: Raven Press. 1993.
82. Schwartzkroin, P.A. Characteristics of CA1 neurons recorded intracellularly in the hippocampal in vitro slice preparation. Brain Res., 85: 423-436, 1975.

83. Schwartzkroin, P.A. Role of the hippocampus in epilepsy. Hippocampus, 4: 239-242, 1994.

84. Schwartzkroin, P.A. The electrophysiology of human brain slices resected from "epileptic" brain tissue. In: Fundamental mechanisms of human Function, edited by J.Engel, Jr., New York: Raven, 145-154., 1987.

85. Sloviter, R.S. On relationship between neuropathology and pathophysiology in the epileptic hippocampus of human and experimental animals. Hippocampus, 4: 250-253, 1994.

86. Sloviter, R.S. Possible functional consequences of synaptic reorganization in the dentate gyrus of kainated-treated rats. Neurosci. Lett., 137: 91-96, 1992.

87. Sommer, W. Rrkrankung des ammonshorns als aetiologisches moment der epilepsie. Arch Psychiatr Nervenkr., 10:631-635, 1880.

88. Sutula, T.; Steward, O. Facilitation of kindling by prior induction of long-term potentiation in the perforant path. Brain Res., 420: 109-117, 1987.

89. Sutula, T.P. Experimental models of temporal lobe epilepsy: new insights from the study of kindling and synaptic reorganization. Epilepsia, 31: s45-s54, 1990.

90. Tasker, J.G.; Peacock, W.J.; Dudek, F.E. Local synaptic circuits and epileptiform activity in slice of neocortex from children with intractable epilepsy. J Neurophysiol, 67: 496507, 1992.

91. Tauck, D.L.; Nadler, J.V. Evidence of functional mossy fiber sprouting in hippocampal formation of kainic acid treated rats. J. Neurosci, 5: 1016-1022, 1985.

92. Traub, R.D.; Jefferys, J.G.R.; Whittington, M.A. Enhanced NMDA conductance can account for epileptiform activity by low $\mathrm{Mg}^{2+}$ in the rat hippocampal slice. J. Physiol., 478: 379393. 1994.

93. Traub, R.D.; Miles, R.; Muller, R.U.; Gulyas, A.I. Functional organization of the hippocampal CA3 region: implications for epilepsy, brain waves and spatial behavior. Network, 3: 465488,1992

94. Traub, R.D.; Miles, R.; Wong, R.K.S. Model of the origin of rhythmic population oscillations in the hippocampal slice. Science, 243: 1319-1325, 1989.

95. Traynelis, S.F.; Dingledine, R. Potassium-induced spontaneous electrographic seizures in the rat hippocampal slice. J. Neurophysiol., 59: 259-276, 1988.

96. Urban, L.; Aitken, P.G.; Crain, B.J.; Friedman, A.H.; Somjen, G. Correlation between function and structure in "epileptic" human hippocampal tissue maintained in vitro. Epilepsia, 34 : 54-60, 1993.

97. Urban, L.; Aitken, P.G.; Friedman, A.; Somjen, G.G. An NMDAmediated component of excitatory synaptic input to the dentate granule cells in "epileptic" human hippocampus studied in vitro. Brain Res., 515: 319-322, 1990.

98. Uruno, K.; O'Connor, M.J.; Masukawa, L.M. Alterations of inhibitory synaptic responses in the dentate gyrus of temporal lobe epileptic patients. Hippocampus, 4: 583-593, 1994

99. Vreugdenhil, M.; Wadman, W.J. Kindling induced long-lasting enhacement of calcium current in hippocampal CA1 area of the rat: relation to calcium-dependent inactivation. Neuroscience, 59: 105-114, 1994.

100. Walther, H.; Lambert, J.D.C.; Jones, R.S.G.; Heinemann, U.; Hamon, B. Epileptiform activity in combined slices of the hippocampal, subiculum and entorhinal cortex during perfusion with low magnesium medium. Neurosci. Lett., 69 : 156-161, 1986.

101. Ward, A.A.; Thomas, L.B. The electrical activity of single units in the cerebral cortex of man. Electroenceph. Clin. Neurophysiol., 7: 135-136, 1955 
102. Warman, E.N.; Durand, D.M.; Yuen, G.L.F. Reconstruction of hippocampal CA1 pyramidal cell electrophysiology by computer simulation. J. Neurophysiol., 71:2033-2045, 1994

103. Willianson, A.; Telfeian, A.E.; Spencer, D.D. Prolonged GABA responses in dentate granule cells in slices isolated from patients with temporal lobe sclerosis. J. Neurophysiol., 74: 378-387, 1995.

104. Wuarin, J.P.; Dudek, E. Electrographic seizures and new recurrent excitatory circuits in the dentate gyrus of hippocampal slices from kainate-treated epileptic rats. Neuroscience, 14:4438-4448, 1996.

105. Wuarin, J.P.; Peacock, W.J.; Dudek, F.E. Single-electrode voltage-clamp analysis of the $\mathrm{N}$-methyl-D-aspartate component of synaptic responses in neocortical slices from children with intractable epilepsy. J Neurophysiol., 67: 4-93. 1992

106. Yaari, Y.; Konnerth, A.; Heinemann, U. Nonsynaptic epileptogenesis in the mammalian hippocampus in vitro. II. Role of extracellular potassium. J. Neurophysiol., 56: 424438, 1986.
107. Yamamoto, $C$.; Mcllwain, $H$. Electrical activities in thin sections from the mammalian brain maintained in chemically-defined media in vitro. J. Neurochem., 13: 1333-1343, 1966.

108. Yamamoto, C. Intracellular study of seizure-like afterdischarges elicited in thin hippocampal sections in vitro. Exp. Neurol., 35: 154-164, 1972.

109. Yeh, G.C.; Bonhaus, D.W.; Nadler, J.V.; McNamara, J.O. Nmethyl-D-aspartate receptor plasticity in kindling: quantitative and qualitative alterations in the $\mathrm{N}$-methyl-D-aspartate receptor-channel complex. Proc Natl Acad Sci. USA, 86: 81578160,1989

110. Zhang, C.L.; Gloveli, T.; Heinemann, U. Effects of NMDA- and AMPA-receptor antagonists on different forms of epileptiform activity in rat temporal cortex slices. Epilepsia, 35(Suppl. 5): s6-9, 1994.

111. Zhou, Q.; Abe, H.; Nowak Jr., T.S. Immunocytochemical and in situ hybridization approaches to the optimization of brain slice preparations. J. Neurosci. Methods, 59: 85-92, 1995. 